\section{Chemokine signaling guides regional patterning of the first embryonic artery}

\author{
Arndt F. Siekmann, ${ }^{1,4,6}$ Clive Standley, ${ }^{2}$ \\ Kevin E. Fogarty, ${ }^{2}$ Scot A. Wolfe, ${ }^{1,3}$ \\ and Nathan D. Lawson ${ }^{1,5}$ \\ ${ }^{1}$ Program in Gene Function and Expression, University of \\ Massachusetts Medical School, Worcester, Massachusetts \\ 01602, USA; ${ }^{2}$ Biomedical Imaging Group, Program in Molecular \\ Medicine, University of Massachusetts Medical School, \\ Worcester, Massachusetts 01602, USA; ${ }^{3}$ Department of \\ Biochemistry and Molecular Pharmacology, University of \\ Massachusetts Medical School, Worcester, Massachusetts \\ 01602, USA
}

The aorta traverses the body, yet little is known about how it is patterned in different anatomical locations. Here, we show that the aorta develops from genetically distinct endothelial cells originating from diverse locations within the embryo. Furthermore, chemokine (C-X$C$ motif) receptor $4 a(c x c r 4 a)$ is restricted to endothelial cells derived from anterior mesoderm, and is required specifically for formation of the lateral aortae. $C x c 112 b$, a cxcr4a ligand, is expressed in endoderm underlying the lateral aortae, and loss of $c x c 112 b$ phenocopies $\operatorname{cxcr} 4 a$ deficiency. These studies reveal unexpected endothelial diversity within the aorta that is necessary to facilitate its regional patterning by local cues.

Supplemental material is available at http://www.genesdev.org.

Received April 21, 2009; revised version accepted August 17, 2009.

The aorta is the first artery to develop in vertebrate embryos and is thought to form largely through vasculogenesis, or the de novo formation of blood vessels (Coffin and Poole 1988; Cleaver and Krieg 1999). In zebrafish embryos, the dorsal aorta (DA) forms as a single vessel in the trunk region of the embryo (Lawson and Weinstein 2002) while in more anterior regions the lateral dorsal aortae (LDA) form as paired vessels on either side of the embryo (Isogai et al. 2001). Recent results have shown that midline structures within the trunk, such as the notochord, are necessary for DA patterning during this process (Fouquet et al. 1997; Sumoy et al. 1997; Cleaver and Krieg 1998). In some cases, the midline serves as a source of angiogenic signals such as vascular endothelial growth factor (Vegf) that promote the migration of

[Keywords: Aorta; zebrafish; zinc finger nuclease; differentiation; patterning; chemokine]

${ }^{4}$ Present address: Max-Planck-Institute for Molecular Biomedicine, Roentgenstr. 20, 48149 Muenster, Germany.

Corresponding authors.

${ }^{5}$ E-MAIL nathan.lawson@umassmed.edu; FAX (508) 856-5460.

${ }^{6}$ E-MAIL arndt.siekmann@mpi-muenster.mpg.de; FAX 49-251-70365-459.

Article is online at http://www.genesdev.org/cgi/doi/10.1101/gad.1813509. arterial endothelial cell progenitors to the midline, where they form the DA (Cleaver and Krieg 1998). Alternatively, the notochord secretes morphogens such as Sonic hedgehog (Shh) that instruct surrounding tissues to express Vegf, which in turn promotes patterning of trunk arteries (Lawson et al. 2002, 2003). This process is facilitated, in part, by the early differentiation of arterial and venous endothelial cell fates that allow distinct cell populations to respond differentially and appropriately to diverse morphogenetic signals (Lawson et al. 2002; Covassin et al. 2006). While zebrafish embryos deficient for Shh or Vegf or lacking the notochord itself display defective DA formation, the anterior vasculature, including the LDA, appears unaffected in these cases (see Supplemental Fig. 1; Stainier et al. 1996; Fouquet et al. 1997; Sumoy et al. 1997; Nasevicius et al. 2000), suggesting the existence of distinct mechanisms that may contribute to the patterning of the aorta in other anatomical locations.

In addition to Vegf, a number of other ligands and their receptors play important roles during the development of the vascular system. Among these are chemokines, which are small (8- to $14-\mathrm{kDa}$ ) vertebrate-specific proteins that can be categorized into four subgroups according to the presence and position of conserved cysteine residues $(\mathrm{C}$, CC, CXC, and CX3C) (Raz and Mahabaleshwar 2009). These secreted proteins were initially identified as important mediators of the immune response (Luster 1998). More recently, chemokines of the CXC class have been implicated in a wide variety of developmental processes, such as primordial germ cell migration, axon growth, lateral line formation, and endoderm development ( $\mathrm{Raz}$ and Mahabaleshwar 2009). In these settings, chemokines regulate the migratory behavior of individual cells or groups of cells. Of interest, mice deficient for either chemokine (C-X-C motif) receptor $4 a$ (cxcr4) or its ligand, cxc112/sdf1, show specific defects in the gut vasculature (Nagasawa et al. 1996; Tachibana et al. 1998), suggesting a role of this ligand/receptor pair in vascular patterning.

In this study, we demonstrate that the developing aorta is comprised of several genetically distinct arterial endothelial cell populations that arise from different anatomical locations. In particular, we find that the zebrafish cxcr4 ortholog cxcr $4 a$ is specifically expressed in arterial endothelial cells derived from anterior but not posterior lateral mesoderm. Embryos deficient for cxcr4a or its ligand, cxcl12b, display defects in anterior LDA formation, while DA formation in the trunk is not affected. Interestingly, $c x c 112 b$ is expressed in the endoderm immediately adjacent to the developing LDA, and embryos lacking endoderm fail to form an LDA. Together, our observations reveal new levels of differentiation within the developing vascular system and implicate this diversity in governing the appropriate response to local extrinsic guidance cues during blood vessel patterning.

\section{Results and Discussion}

To better understand the mechanisms that contribute to the formation of the anterior aorta, we performed twophoton time-lapse analysis of $T g(k d r l: e g f p)^{1 a 116}$ embryos in which endothelial cells are labeled with enhanced green fluorescent protein (egfp) (Choi et al. 2007). These movies revealed that formation of the LDA occurs in 
a bidirectional manner: Starting at $14 \mathrm{~h}$ post-fertilization (hpf), we observed endothelial cells from the anterior lateral plate mesoderm migrating posteriorly (Fig. 1A, anterior LDA, cells labeled in red), while at the same time, endothelial cells from posterior regions of the embryo started to migrate anteriorly (Fig. 1A, posterior LDA, cells labeled in green). These cells continued to migrate toward each other, finally fusing to form a continuous vessel by $20.5 \mathrm{hpf}$ (Fig. 1B-F; see also Supplemental Movies 1, 2). At $\sim 17 \mathrm{hpf}$, venous endothelial cells also started to sprout parallel to the LDA in a similar bidirectional manner, completing the formation of the major head vein, called the primordial hindbrain channel (PHBC), by 24 hpf (Fig. 1C-F, arrowheads, PHBC labeled in blue). While the LDAs formed via angiogenic sprouting, the single axial DA in the embryonic trunk formed via the medial migration of endothelial cells from the adjacent lateral plate mesoderm, connecting to the LDAs to form a Y-shaped structure (Supplemental Figure S2A-F; Supplemental Movies 3,4). Thus, based on these observations, we can delineate at least three different endothelial cell populations that contribute to the primitive aorta: (1) cells from the anterior lateral mesoderm that contribute to the anterior LDA, (2) cells from the posterior lateral mesoderm that contribute to the posterior LDA, and (3) posterior lateral mesoderm endothelial cells that migrate medially to form the DA /see also Figs. $1 \mathrm{~F}, 2 \mathrm{I})$.

We reasoned that these different migratory behaviors might correspond to genetic differences within aortic endothelial cells. To determine if this was the case, we searched an online expression pattern database available through the Zebrafish Information Network (http:// www.zfin.org) for genes expressed specifically in arterial endothelial cells. We subsequently performed wholemount in situ hybridization with candidate genes at different embryonic stages to more closely monitor their expression pattern within the developing aorta. We found several genes that were expressed exclusively in individual domains delineated by our time-lapse studies. We observed that $\mathrm{cxcr} 4 a$ displays strikingly specific expression in arterial endothelial cells within the anterior, but not posterior, lateral mesoderm starting as early as the
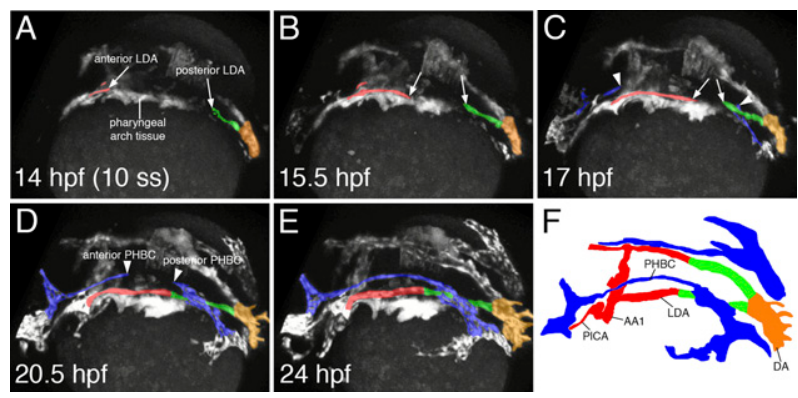

Figure 1. Two-photon time lapse of LDA formation in live zebrafish embryos. $(A-F)$ Dorsolateral view of LDA formation. Anterior LDA is pseudocolored in red, posterior LDA is shown in green, axial $\mathrm{DA}$ is indicated by orange, and the PHBC is shown in blue. Arrows mark migrating cells of the LDA, which forms in a bidirectional manner. Arrowheads indicate similarly migrating PHBC cells. $(F)$ Labeled camera lucida image indicating position of vessels in $E$. (DA) Dorsal aorta; (LDA) lateral dorsal aorta; (PHBC) primordial hindbrain channel; (PICA) primitive internal carotid artery; (AA1) aortic arch 1.

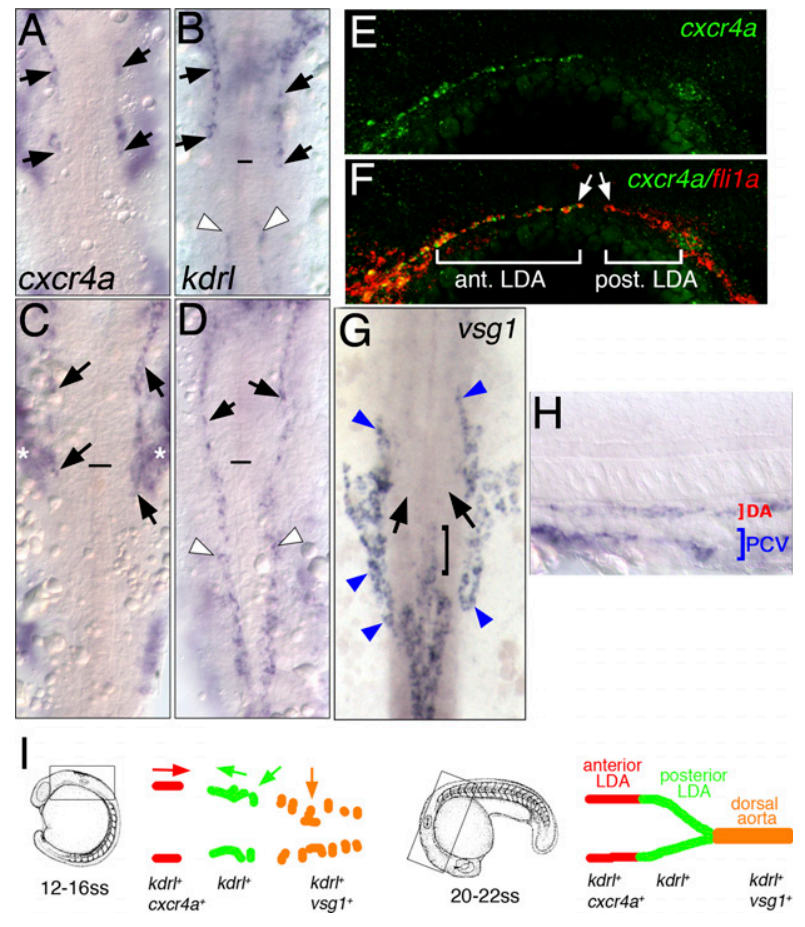

Figure 2. LDA progenitors are genetically distinct. $(A-H)$ In situ hybridization with indicated markers. $(A-D, G)$ Dorsal view, anterior is up. $(E, F, H)$ Lateral view, anterior to the left, dorsal is up. $(A-D)$ Expression of $\operatorname{cxcr} 4 a(A, C)$ and $k d r l(B, D)$ at the 18-somite stage and the 22-somite stage, respectively. Anterior LDA (black arrows) and posterior LDA (white arrowheads) are indicated. Black line marks the anterior end of the notochord. Asterisks in $C$ mark expression of $c x c r 4 a$ in pharyngeal arch tissue. $(E, F)$ Confocal images of 22-somitestage embryos following double-fluorescent in situ hybridization. Endothelial cells are labeled by expression of flila in red and cxcr $4 a$ in green. $(G, H)$ Expression of $v s g 1$ at the 22-somite stage. $(G)$ Dorsal view. $(H)$ Lateral view. $(G)$ Black arrows mark posterior LDA, which does not express vsg1. Bracket marks anterior end of DA. Blue arrowheads mark venous cells. (I) Schematic drawing of DA domains delineated by different migratory behaviors and gene expression profiles at the 18-somite stage and the 22-somite stage.

12-somite stage (15 hpf) (Supplemental Fig. S3). In contrast, the duplicate $c x c r 4 b$ gene does not appear to be expressed in endothelial cells (ZDB-PUB-010810-1, http://www.zfin. org). During the convergent migration of the anterior and posterior LDA branches (17-18 hpf), cxcr4a expression can be observed exclusively in the anterior endothelial cell population (Fig. 2A; Supplemental Fig. S3), while the zebrafish vegf receptor- 2 ortholog $k d r l$ is expressed in both anterior and posterior branches (Fig. 2B). We did not detect cxcr $4 a$ expression in venous endothelial cells at this stage. Expression of cxcr $4 a$ is similarly restricted to the anterior domain of the LDA at the 22-somite stage (Fig. 2C; Supplemental Fig. S3), at which time these blood vessels have fused and are labeled throughout by kdrl (Fig. 2D). By $24 \mathrm{hpf}, \operatorname{cxcr} 4 a$ expression is apparent throughout the LDA and in the axial DA within the trunk (Supplemental Fig. S3). More detailed analysis using two-color fluorescence in situ hybridization demonstrated coexpression of cxcr4a and the pan-endothelial marker fli1 $a$ in the anterior, but not posterior, domains of the LDA (Fig. 2E,F). In contrast, we observed expression of $v s g 1$, a zebrafish ortholog of plasmalemma vesicle-associated protein (plvap), in the 
DA, but not in the branches of the LDA at the 18-somite stage and the 22-somite stage (Fig. 2G,H); vsg1 expression was also observed in venous endothelial cells at this stage (Fig. 2G,H). Together with our time-lapse analyses, these results demonstrate that the aorta is comprised of genetically distinct arterial endothelial cells that arise from different anatomical origins (Fig. 2I).

Chemokine signaling is important for directing cell migration in a variety of tissue types (Raz and Mahabaleshwar 2009). Therefore, we reasoned that cxcr4a might play a crucial role in guiding the migration of arterial endothelial cells during formation of the LDA. To determine if this was the case, we used zinc finger nucleases (ZFNs) to introduce a targeted deletion within exon 2 of $c x c r 4 a$ (Fig. 3A). We constructed individual ZFN monomers by modular assembly (see the Supplemental Material) and introduced them together into zebrafish embryos via mRNA coinjection at the one-cell stage. We subsequently identified mosaic founders by assaying for loss of a DdeI restriction site within the target sequence (Fig. 3A,B) in progeny embryos. F2 families of heterozygous carriers derived from initial founders were used to generate $c x c r 4 a$ mutant embryos. The um20 allele used for subsequent analysis causes an in-frame 5-amino-acid deletion within the second transmembrane domain of the Cxcr4a receptor that eliminates its membrane localization (Fig. 3B; Supplemental Fig. S4). Compared with their wild-type siblings, $c x c r 4 a^{u m 20}$ mutants displayed mild cardiac edema associated with a delay in the onset of circulation between 24 and 32 hpf (Fig. 3C,F; data not shown), while their general morphology was otherwise indistinguishable (Fig. 3C,F; Supplemental Fig. S5). By day 2 of development, we observed that approximately half of $\mathrm{cxcr} 4 a^{\text {um20 }}$ mutant embryos failed to exhibit active circulation (Supplemental Table 1). We further observed endoderm defects, which have been reported in zebrafish embryos injected with a morpholino targeting the endogenous cxcr $4 a$ start codon (Mizoguchi et al. 2008; Nair and Schilling 2008). In particular, we noted precocious endoderm migration during gastrulation and ectopic clusters of insulin-positive pancreatic islet cells at day 2 of development (Supplemental Fig. S6). As with the observed circulatory defects, abnormal islet cell localization was partially penetrant (Supplemental Table 1). Importantly, the partially penetrant circulatory and pancreas phenotypes could be observed independently in some cxcr4am20 mutant embryos (Supplemental Table 1), suggesting an autonomous role for $\mathrm{cxcr} 4 a$ in each tissue type.

To determine the primary vascular defects caused by loss of $c x c r 4 a$ function, we observed blood vessel morphology in wild-type and cxcr4a-deficient embryos bearing the $\mathrm{Tg}(\mathrm{kdrl}: e g f p)^{1 a 116}$ transgene. At $32 \mathrm{hpf}$, wild-type siblings displayed completely formed LDA and PHBC, as well as a normal vascular network in the trunk (Fig. $3 \mathrm{D}, \mathrm{E})$. In contrast, we observed incomplete formation of the LDA in cxcr4aum20 mutant embryos (Fig. 3G) or embryos injected with a cxcr4a morpholino (Supplemental Fig. S7; Supplemental Tables 2, 3). Formation of the PHBC and the major trunk vessels was normal in cxcr4adeficient embryos at this developmental stage (Fig. 3G, H; Supplemental Fig. S7). As mentioned above, cxcr4a deficiency causes endoderm defects that may affect LDA development. However, transplantation of wild-type endoderm into cxcr $4 a^{\text {um20 }}$ mutant embryos failed to rescue LDA formation (Supplemental Fig. S8; Supplemental

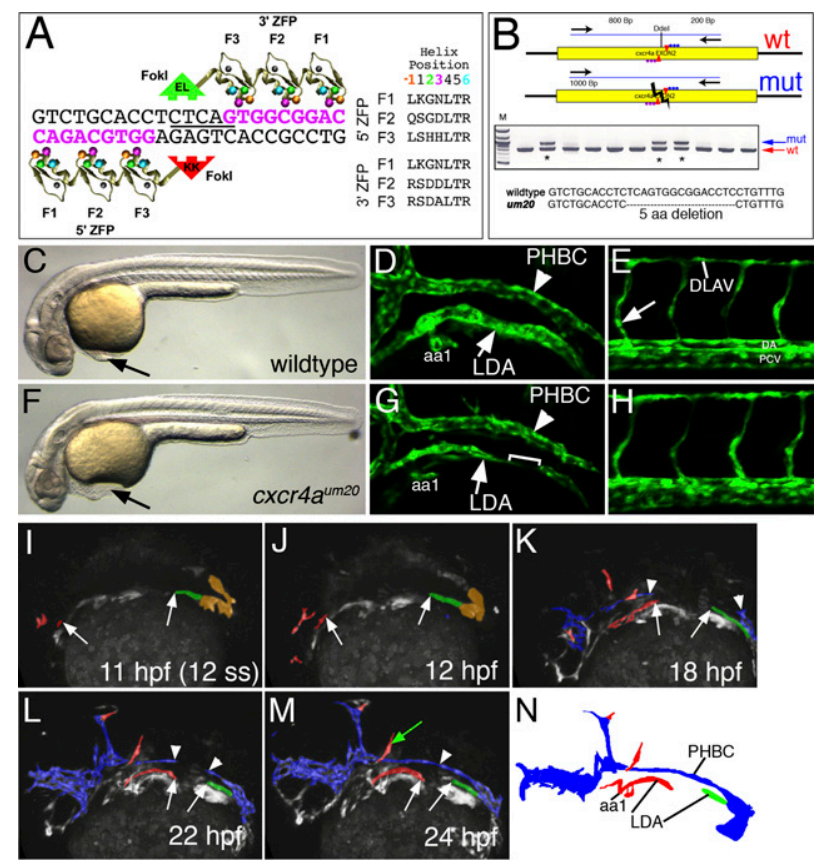

Figure 3. LDA formation defects in cxcr4a-deficient embryos. $(A)$ Schematic depicting ZFN monomers designed against $\mathrm{cxcr} 4 a$ exon 2 target site. Nucleotides in pink represent the recognition elements. The DdeI site within the spacer region is underlined. Recognition helices for each finger are indicated. $(B)$ PCR genotyping analysis of embryos derived from putative founder fish. Failure to digest with DdeI is indicative of a possible target site deletion. An asterisk marks genotypes that bear mutagenic lesions. $(C, F)$ Transmitted light images of wild-type $(C)$ and $\operatorname{cxcr} 4 a$ mutant embryos $(F)$ at the 32-hpf stage. Lateral views, dorsal is up, anterior is to the left. $(D, E, G, H)$ Confocal images showing anterior regions $(D, G)$ or posterior regions $(E, H)$ of wild-type $(D, E)$ or cxcr $4 a$ mutant $(G, H)$ embryos. (LDA) Lateral dorsal aorta; (PHBC) primordial hindbrain channel; (aal) aortic arch1. Bracket in $G$ indicates a gap within LDA. (DLAV) Dorsal longitudinal anastomotic vessel; (DA) dorsal aorta; (PCV) posterior cardinal vein. The arrow in $E$ indicates the intersegmental blood vessel sprout. (I-M) Stills of two-photon time-lapse (see Supplemental Movie 5) showing LDA formation in $\mathrm{cxcr}_{4} a^{u m 20}$ mutant embryos. Lateral views, anterior is to the left. Time points are indicated. Red labels arterial LDA cells, green indicates posterior LDA cells, and blue labels venous endothelial cells. Arrows mark anterior and posterior LDA, while arrowheads mark the forming PHBC. The green arrow in $M$ marks ectopically located endothelial cells. (N) Camera lucida drawing of embryo in $M$.

Table 5), indicating that the observed vascular defects were not a result of abnormal endoderm patterning.

To further investigate defects in LDA formation, we performed two-photon time-lapse analysis of $\mathrm{Tg}(\mathrm{k} d r \mathrm{r}$ : egfp $)^{1 a 116}{ }_{;} \mathrm{cxcr} 4 a^{\text {um20 }}$ mutant embryos. Between 14 and 24 hpf, we observed that initial sprouts of the anterior and posterior origins of the LDA formed normally, but failed to connect as in wild-type embryos (Fig. 3I-N; Supplemental Movie 5). Time-lapse movies of cxcr4a mutant embryos up to $36 \mathrm{hpf}$ revealed failure to connect the branches of the LDA, suggesting that this defect was not due to developmental delay (Supplemental Movie 6). We noted similar LDA defects in time-lapse movies of cxcr4a morpholino-injected embryos (Supplemental Movie 7). In all cases, formation of the PHBC and trunk blood vessels was unaffected by the loss of $c x c r 4 a$ function. Interestingly, we observed ectopic migration of arterial endothelial 
cells and inappropriate arteriovenous connections in embryos lacking cxcr4a function (Supplemental Movie 5; Fig. 3M, green arrow; Supplemental Movie 6, respectively). This cell behavior was not found in wild-type embryos. Taken together, our observations indicate that cxcr $4 a$ is specifically required to guide a subset of arterial endothelial cells during formation of the anterior LDA.

The zebrafish genome encodes two duplicate Cxcr4 ligands, referred to as $c x c 112 a$ and $c x c 112 b$. While $c x c 112 a$ acts primarily through $c x c r 4 b$ to promote sensory axon pathfinding and germ cell migration (Doitsidou et al. 2002; Miyasaka et al. 2007), cxcl12b and $c x c r 4 a$ are required for endoderm formation (Mizoguchi et al. 2008; Nair and Schilling 2008). Given the importance of $c x c r 4 a$ for LDA formation, we asked if $c x c 112 b$ might be involved in LDA patterning. In order to address this question, we first analyzed the expression pattern of $c x c 112 b$ during LDA formation. Whole-mount in situ hybridization revealed that $c x c 112 b$ is expressed in tissue underlying the arterial endothelial cells that will comprise the LDA before and after their migration (Fig. 4A,C-E; Supplemental Fig. S9). This expression was severely reduced in embryos injected with a morpholino targeting sox32 that lack the endoderm (Fig. 4B; Chung and Stainier 2008), but was normal in cxcr4a $a^{\text {um20 }}$ mutant embryos (data not shown). We next addressed the effect of loss of either $c x c 112 b$ or the endoderm itself on vascular func-

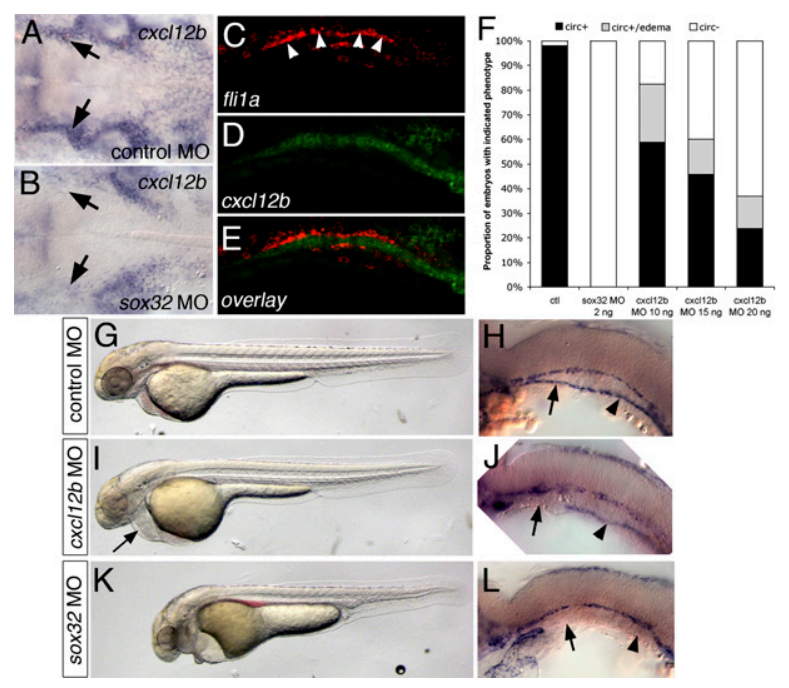

Figure 4. Endoderm-derived $c x c 112 b$ is required for LDA formation. $(A, B)$ In situ hybridization at 24 hpf showing $c x c 112 b$ expression in tissue underlying the LDA (arrows). (B) Loss of endoderm in sox32 morpholino-injected embryos leads to severe reduction in $c x c l 12 b$ expression. Dorsal view, anterior is to the left. $(C-E)$ Confocal micrographs of 22-hpf embryo subjected to double-fluorescent whole-mount in situ hybridization, lateral view, anterior is to the left, dorsal is up. (C) fli1a expression, marking the LDA. (D) $c x c 112 b$ expression. (E) Overlay; fli1a is red, $c x c 112 b$ is green. $(F)$ Influence of loss of endoderm or $c x c 112 b$ on circulation. (ctl) Control morpholino-injected; $\left(\operatorname{circ}^{+}\right)$embryos with circulation; (circ) embryos without circulation. $(G-L)$ Vascular defects in embryos lacking endoderm or cxcl12b. $(G, I, K)$ Bright-field views at $32 \mathrm{hpf}$, anterior is to the left, dorsal is up. $(H, J, L)$ In situ hybridization for kdrl at $24 \mathrm{hpf}$. Black arrows indicate anterior LDA, arrowheads indicate posterior LDA. $(G, H)$ Embryos injected with control morpholino. $(I, L)$ Embryos injected with $15 \mathrm{ng}$ of $c x c 112 b$ MO. $(K, L)$ Embryos injected with $2 \mathrm{ng}$ of sox32 morpholino. Arrows mark the anterior LDA, arrowheads mark the posterior LDA. tion and LDA formation. In comparison with control morpholino-injected embryos (Fig. 4F-H), embryos injected with a morpholino targeting $c x c 112 b$ displayed complete loss of circulation, or slow circulation associated with pericardial edema (Fig. 4F,I, arrow; Supplemental Table 4), although heart morphology appeared grossly normal (data not shown). Similar to $\operatorname{cxcr} 4 a^{\text {um20 }}$ mutant embryos, $c x c 112 b$-deficient embryos also displayed defects in LDA formation (Fig. 4J; Supplemental Movie 8; Supplemental Fig. S7; Supplemental Tables 2, 3). In contrast, embryos injected with a morpholino targeting the closely related $\operatorname{cxcl12a}$ paralog displayed normal circulation and LDA formation (Supplemental Tables 2, 3; data not shown). The effect on LDA formation was even more pronounced in embryos injected with sox32 morpholino (Fig. 4F,K,L; Supplemental Fig. S7). Here, both anterior and posterior branches of the LDA failed to migrate (Fig. 4L), and injected embryos never established circulation (Fig. 4F; Supplemental Movie 9; Supplemental Fig. S7). These observations indicate that endoderm-derived $c x c 112 b$ serves as an important guidance cue to direct migration of cxcr $4 a$-positive arterial endothelial cells during formation of the LDA.

Proper assembly of a mature circulatory system is essential for cardiovascular function during embryonic development and postnatal life. An important step in this process is the appropriate response of endothelial cells to extrinsic guidance and growth factor cues in the developing embryo. In our present study, we show that aorta development entails coordinate migration of distinct populations of arterial endothelial progenitors. We provide further evidence that this process is facilitated by an unexpected molecular diversity within these cells. In this case, restricted cxcr $4 a$ expression directs a subset of arterial endothelial cells from the anterior lateral mesoderm to follow an endoderm-derived chemokine that guides formation of the LDA. Although defects in LDA formation have not been described in mouse embryos deficient for either cxcr4 or $c x c 112 b$, defects in other endoderm-associated blood vessels, such as those in the gut, are evident (Nagasawa et al. 1996; Tachibana et al. 1998), suggesting a possible conserved role for this pathway. Furthermore, mouse embryos with deficient endodermal differentiation display specific defects in anterior DA patterning (Sakamoto et al. 2007), while posterior aorta development is not affected, suggesting that genetic differences between anterior and posterior regions of the LDA might also exist in mice.

Interestingly, cxcr $4 a$ and $c x c 112 b$ are dispensable for trunk blood vessel formation in zebrafish, which instead is highly dependent on Vegf signaling (Lawson et al. 2003). In this setting, different Vegf receptors are restricted to distinct endothelial cell populations and are specifically required for trunk artery patterning (Covassin et al. 2006; Siekmann and Lawson 2007), analogous to the role for $c x c r 4 a$ in patterning the anterior vasculature. Similarly, PHBC development proceeds normally in the absence of endoderm or cxcr4a and instead requires Vegfc signaling through Flt4, which is largely restricted to venous endothelial cells (Covassin et al. 2006). Thus, endothelial cell differentiation appears to play an essential role in vascular morphogenesis by establishing the appropriate complement of signaling molecules that enable some, but not all, cells to respond to a particular extrinsic factor. This differentiation process enables the 
coordinated migration, coalescence, and sprouting of endothelial cells throughout the diverse extracellular milieu in distinct anatomical locations within the embryo. Ultimately, the intimate connection between these two processes-differentiation and morphogenesis-enables the formation of an appropriately patterned and patent vascular system that can bear circulatory flow.

\section{Materials and methods}

\section{Zebrafish strains}

Zebrafish were maintained as described previously (Westerfield 1993). The establishment and characterization of the $T g(k d r l: e g f p)^{1 a 116}$ transgenic line have been described elsewhere (Choi et al. 2007). The $n t 1^{b 160}$ mutant zebrafish (Halpern et al. 1993) were obtained from Erez Raz.

\section{Generation of $\mathrm{cxcr} \mathrm{a}^{\mathrm{um} 20}$ mutant zebrafish}

ZFNs specific for exon2 of cxcr4a were designed as described in the Supplemental Material. Generation and identification of mutants were essentially carried out as described previously (Meng et al. 2008). Primers for genotyping were cxcr4a FWD1, 5' -CCAACTTTGAGGTCCCGTGTG ATG-3'; and cxcr4a REV1, 5'-CTGTGGACACGGATGACATTCCTG-3' PCR products were digested using DdeI. We identified several founder fish that carried different genomic lesions. From one of these, we established the $\operatorname{cxcr} 4 a^{u m 20}$ line, containing a 5-amino-acid in-frame deletion from position 87 to 91 in the $c x c r 4 a$ ORF.

\section{Time-lapse, two-photon, and confocal microscopy}

Confocal microscopy of wild-type and cxcr $4 a^{u m 20}$ embryos was carried out as described previously (Covassin et al. 2006). Time lapses were essentially carried out as described (Lawson and Weinstein 2002); however, we used a two-photon microscope to reduce photobleaching. Live embryos were mounted in low-melting-point agarose in tissue culture dishes at the 10-somite stage and imaged with Bio-Rad Radiance MP2100 two-photon microscope, consisting of a Spectra Physics laser system (MillenniaV, 5-W, 532-nm diode-pumped laser, which pumps a mode locked Tsunami Ti:sapphire laser). We used a BX50WI Olympus upright microscope with an Olympus XLUMPlanFl 20×, NA 0.95 water-dipping objective. Both the objective and the sample stage were temperaturecontrolled to $28^{\circ} \mathrm{C}$. The two-photon excitation wavelength used in these experiments was $830 \mathrm{~nm}$, and an eGFP/Red emission filter cube from Chroma technology was used giving an observation bandpass of 485-535 $\mathrm{nm}$. To prevent movement of the embryo, medium was supplemented with $0.1 \%$ Tricaine. Pigmentation of the embryos was inhibited by adding Phenylthiourea to a final concentration of $0.003 \%$. Confocal stacks and two-photon movies were assembled using Imaris Software (Bitplane). Movies were labeled using Flash Player (Macromedia).

\section{Morpholinos and whole-mount in situ hybridization}

Morpholinos for cxcr4a (MORPH 1667, 5' -AGACGATGTGTTCGTAAT AAGCCAT-3'), cxcl12b (MORPH 1736, 5'-CGCTACTACTTTGCTA TCCATGCC-3'), cxc112a (MORPH 1176, 5'-ACTTTGAGATCCATG TTTGGCAGTG-3'), and sox32 (MORPH 0008, 5'-CGGTCGAGAT ACATGCTGTTTTGCG-3') were initially obtained from Open Biosystems. Sequence-identical morpholinos were then reordered from GeneTools. All morpholinos were dissolved in $\mathrm{H}_{2} \mathrm{O}$. The morpholinos for cxcr4a and sox32 have been published previously (Hollway et al. 2007, Chung and Stainier 2008). Morpholinos for $c x c 112 a$ and $c x c 112 b$ differ in one nucleotide from previously published morpholinos (Miyasaka et al. 2007). As a control, we injected standard control morpholinos (5'-CCTC TTACCTCAGTTACAATTTATA-3') into sibling embryos. Whole-mount in situ hybridization and imaging for $k d r l$ were carried out as described (Lawson et al. 2002). A plasmid with zebrafish cxcr4a was kindly provided by Beth Roman (University of Pittsburgh). The probe for $c x c 112 b$ was provided by Nobuhiko Miyasaka (RIKEN Brain Sciene Instiute, Saitama) (Miyasaka et al. 2007). To generate the probe for vsg1, we obtained an EST for vsg1 from Open Biosystems (NAA06F04), digested it with SmaI, and used Sp6 to generate the riboprobe. Double-fluorescent in situ hybridization was carried out as described previously (Julich et al. 2005). The probe for fli1 was described previously (Lawson et al. 2001).

\section{Acknowledgments}

We thank Ralf Adams and Rui Benedito for critical reading of the manuscript. We thank John Polli and Mike Kacergis for excellent fish care and maintenance. We are also grateful to Erez Raz for providing the $n t l^{b 160}$ mutant zebrafish and Jau-Nian Chen for Tg(kdrl:egfp $)^{1 a 116}$ transgenic zebrafish. We are grateful to Sreelaja Nair and Tom Shilling for providing the TARAM A plasmid. We also thank members of the Lawson and Wolfe laboratories for helpful discussions, and Julie Zhu for the statistical analysis. This work was supported in part by R01HL079266 awarded to N.D.L. and R01HL093766 awarded to N.D.L. and S.A.W. from the National Heart, Lung, and Blood Institute, NIH. C.S. and K.E.F. were supported by P30 DK032520 from the National Institute of Diabetes and Digestive and Kidney Disease.

\section{References}

Choi J, Dong L, Ahn J, Dao D, Hammerschmidt M, Chen JN. 2007. FoxH1 negatively modulates flk1 gene expression and vascular formation in zebrafish. Dev Biol 304: 735-744

Chung WS, Stainier DY. 2008. Intra-endodermal interactions are required for pancreatic $\beta$ cell induction. Dev Cell 14: $582-593$.

Cleaver O, Krieg PA. 1998. VEGF mediates angioblast migration during development of the dorsal aorta in Xenopus. Development 125: 3905-3914.

Cleaver O, Krieg PA. 1999. Molecular mechanisms of vascular development. In Heart development (eds. RP Harvey and N Rosenthal), pp. 221-252. Academic Press, San Diego, CA.

Coffin JD, Poole TJ. 1988. Embryonic vascular development: Immunohistochemical identification of the origin and subsequent morphogenesis of the major vessel primordia in quail embryos. Development 102: $735-748$

Covassin LD, Villefranc JA, Kacergis MC, Weinstein BM, Lawson ND. 2006. Distinct genetic interactions between multiple Vegf receptors are required for development of different blood vessel types in zebrafish. Proc Natl Acad Sci 103: 6554-6559.

Doitsidou M, Reichman-Fried M, Stebler J, Koprunner M, Dorries J, Meyer D, Esguerra CV, Leung T, Raz E. 2002. Guidance of primordial germ cell migration by the chemokine SDF-1. Cell 111: 647-659.

Fouquet B, Weinstein BM, Serluca FC, Fishman MC. 1997. Vessel patterning in the embryo of the zebrafish: Guidance by notochord. Dev Biol 183: 37-48.

Halpern ME, Ho RK, Walker C, Kimmel CB. 1993. Induction of muscle pioneers and floor plate is distinguished by the zebrafish no tail mutation. Cell 75: 99-111.

Hollway GE, Bryson-Richardson RJ, Berger S, Cole NJ, Hall TE, Currie PD. 2007. Whole-somite rotation generates muscle progenitor cell compartments in the developing zebrafish embryo. Dev Cell 12: 207-219.

Isogai S, Horiguchi M, Weinstein BM. 2001. The vascular anatomy of the developing zebrafish: An atlas of embryonic and early larval development. Dev Biol 230: 278-301.

Julich D, Hwee Lim C, Round J, Nicolaije C, Schroeder J, Davies A, Geisler R, Lewis J, Jiang YJ, Holley SA. 2005. beamter $/ \delta \mathrm{C}$ and the role of Notch ligands in the zebrafish somite segmentation, hindbrain neurogenesis and hypochord differentiation. Dev Biol 286: 391-404.

Lawson ND, Weinstein BM. 2002. In vivo imaging of embryonic vascular development using transgenic zebrafish. Dev Biol 248: 307-318.

Lawson ND, Scheer N, Pham VN, Kim CH, Chitnis AB, Campos-Ortega JA, Weinstein BM. 2001. Notch signaling is required for arterialvenous differentiation during embryonic vascular development. $D e$ velopment 128: 3675-3683.

Lawson ND, Vogel AM, Weinstein BM. 2002. sonic hedgehog and vascular endothelial growth factor act upstream of the Notch pathway during arterial endothelial differentiation. Dev Cell 3: 127-136.

Lawson ND, Mugford JW, Diamond BA, Weinstein BM. 2003. phospholipase $C \gamma-1$ is required downstream of vascular endothelial growth factor during arterial development. Genes \& Dev 17: 1346-1351.

Luster AD. 1998. Chemokines-chemotactic cytokines that mediate inflammation. $N$ Engl J Med 338: 436-445. 
Meng X, Noyes MB, Zhu LJ, Lawson ND, Wolfe SA. 2008. Targeted gene inactivation in zebrafish using engineered zinc-finger nucleases. Nat Biotechnol 26: 695-701.

Miyasaka N, Knaut H, Yoshihara Y. 2007. Cxcl12/Cxcr4 chemokine signaling is required for placode assembly and sensory axon pathfinding in the zebrafish olfactory system. Development 134: 2459-2468.

Mizoguchi T, Verkade H, Heath JK, Kuroiwa A, Kikuchi Y. 2008. Sdf1/ Cxcr4 signaling controls the dorsal migration of endodermal cells during zebrafish gastrulation. Development 135: 2521-2529.

Nagasawa T, Hirota S, Tachibana K, Takakura N, Nishikawa S, Kitamura Y, Yoshida N, Kikutani H, Kishimoto T. 1996. Defects of B-cell lymphopoiesis and bone-marrow myelopoiesis in mice lacking the CXC chemokine PBSF/SDF-1. Nature 382: 635-638.

Nair S, Schilling TF. 2008. Chemokine signaling controls endodermal migration during zebrafish gastrulation. Science 322: 89-92.

Nasevicius A, Larson I, Ekker SC. 2000. Distinct requirements for zebrafish angiogenesis revealed by a VEGF-A morphant. Yeast 17: 294-301.

Raz E, Mahabaleshwar H. 2009. Chemokine signaling in embryonic cell migration: A fisheye view. Development 136: 1223-1229.

Sakamoto Y, Hara K, Kanai-Azuma M, Matsui T, Miura Y, Tsunekawa N, Kurohmaru M, Saijoh Y, Koopman P, Kanai Y. 2007. Redundant roles of Sox17 and Sox18 in early cardiovascular development of mouse embryos. Biochem Biophys Res Commun 360: 539-544.

Siekmann AF, Lawson ND. 2007. Notch signalling limits angiogenic cell behaviour in developing zebrafish arteries. Nature 445: 781-784.

Stainier DY, Fouquet B, Chen JN, Warren KS, Weinstein BM, Meiler SE, Mohideen MA, Neuhauss SC, Solnica-Krezel L, Schier AF, et al. 1996. Mutations affecting the formation and function of the cardiovascular system in the zebrafish embryo. Development 123: 285-292.

Sumoy L, Keasey JB, Dittman TD, Kimelman D. 1997. A role for notochord in axial vascular development revealed by analysis of phenotype and the expression of VEGR-2 in zebrafish flh and ntl mutant embryos. Mech Dev 63: 15-27.

Tachibana K, Hirota S, Iizasa H, Yoshida H, Kawabata K, Kataoka Y, Kitamura Y, Matsushima K, Yoshida N, Nishikawa S, et al. 1998. The chemokine receptor CXCR4 is essential for vascularization of the gastrointestinal tract. Nature 393: 591-594.

Westerfield M. 1993. The zebrafish book. University of Oregon Press, Eugene, OR. 


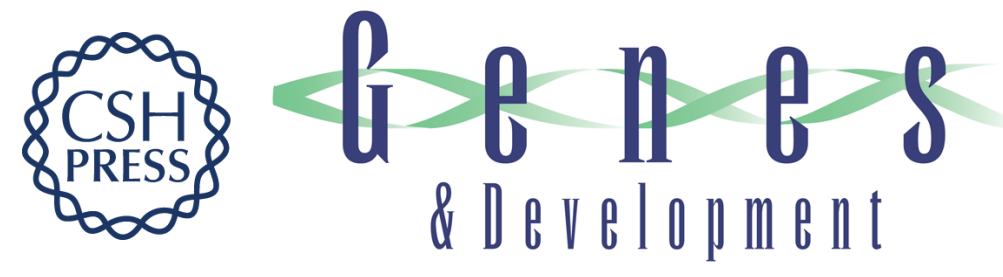

\section{Chemokine signaling guides regional patterning of the first embryonic artery}

Arndt F. Siekmann, Clive Standley, Kevin E. Fogarty, et al.

Genes Dev. 2009, 23:

Access the most recent version at doi:10.1101/gad.1813509

\section{Supplemental http://genesdev.cshlp.org/content/suppl/2009/10/02/23.19.2272.DC1 Material}

References This article cites 28 articles, 10 of which can be accessed free at: http://genesdev.cshlp.org/content/23/19/2272.full.html\#ref-list-1

\section{License}

Email Alerting

Service

Receive free email alerts when new articles cite this article - sign up in the box at the top right corner of the article or click here.

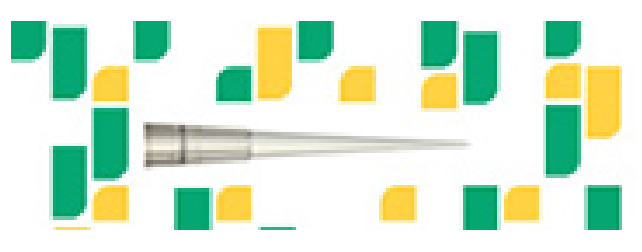

Focused on your science. 